\title{
Author Correction: Are COVID-19 vaccines safe in pregnancy?
}

\section{Victoria Male (iD}

Correction to: Nature Reviews Immunology https://doi.org/10.1038/s41577-021-00525-y, published online 03 March 2021.

There was an error in the third paragraph of text of this Comment article, which incorrectly stated that " 53 pregnancies occurred across the trials of the three vaccines that have so far been approved in the UK"; the actual number of pregnancies is 57 and this has now been corrected online.

https://doi.org/10.1038/s41577-021-00533-y I Published online 12 March 2021

(๑) Springer Nature Limited 2021 